INPLASY

PROTOCOL

To cite: Gao et al. Association between phthalate exposure and insulin resistance: $A$ systematic review and metaanalysis. Inplasy protocol 202140026. doi:

10.37766/inplasy2021.4.0026

Received: 06 April 2021

Published: 06 April 2021

Corresponding author: Hui Gao

gh20190130@163.com

Author Affiliation:

Department of Pediatrics, the

First Affiliated Hospital of

Anhui Medical University

Support: No. 2020kj02;

No.2020xkj161.

Review Stage at time of this submission: Data analysis.

Conflicts of interest:

None declared.

\section{Association between phthalate exposure and insulin resistance: A systematic review and meta-analysis}

\author{
Gao, $\mathrm{H}^{1}$; Chen, D2; Zang, $\mathrm{M}^{3}$.
}

Review question / Objective: The inclusion and exclusion criteria following the PECOS (Population, Exposure, Comparison, Outcome, Study design). Inclusion criteria were the following: Population: human; Exposure: all phthalates or phthalate metabolites exposure; Comparison: lower levels of exposure group, or different levels of target outcome group, or no target disease control group; Outcome: insulin resistance, insulin sensitivity; Study design: all observational studies including cohort study, case-control study and crosssectional survey. Articles were excluded according the following criteria: Population: not human; Exposure: not phthalate or its metabolite exposure; Outcome: any other outcome outside the scope of this review. Study design: review, systematic review, meta-analysis and comments, etc.

INPLASY registration number: This protocol was registered with the International Platform of Registered Systematic Review and Meta-Analysis Protocols (INPLASY) on 06 April 2021 and was last updated on 06 April 2021 (registration number INPLASY202140026).

\section{INTRODUCTION}

Review question / Objective: The inclusion and exclusion criteria following the PECOS (Population, Exposure, Comparison, Outcome, Study design). Inclusion criteria were the following: Population: human; Exposure: all phthalates or phthalate metabolites exposure; Comparison: lower levels of exposure group, or different levels of target outcome group, or no target disease control group; Outcome: insulin resistance, insulin sensitivity; Study design: all observational studies including cohort study, case-control study and crosssectional survey. Articles were excluded 
according the following criteria: Population: not human; Exposure: not phthalate or its metabolite exposure; Outcome: any other outcome outside the scope of this review. Study design: review, systematic review, meta-analysis and comments, etc.

Condition being studied: Association between phthalate exposure and insulin resistance.

\section{METHODS}

Search strategy: The systematic literature search was performed in MEDLINE (accessed through PubMed), Web of Science, and CNKI (Chinese National Knowledge Infrastructure) until March 2021. The following search terms were used in combination: "phthalate" or "phthalic acid ester" or "endocrine disruptor" and their variants, with the words "insulin resistance", "insulin sensitivity", "pre-diabetic state", "hyperinsulinemia", "glucose intolerance", "diabetes", "metabolic syndrome". We only considered human studies. Additional relevant studies were obtained by screening and searching references.

\section{Participant or population: All people.}

Intervention: Phthalate exposure.

Comparator: Lower levels of exposure group, or different levels of target outcome group, or no target disease control group.

Study designs to be included: All observational studies including cohort study, case-control study and crosssectional survey.

Eligibility criteria: Inclusion criteria were the following: Population: human; Exposure: all phthalates or phthalate metabolites exposure; Comparison: lower levels of exposure group, or different levels of target outcome group, or no target disease control group; Outcome: insulin resistance, insulin sensitivity; Study design: all observational studies including cohort study, case-control study and crosssectional survey.
Information sources: The systematic literature search was performed in MEDLINE (accessed through PubMed), Web of Science, and CNKI (Chinese National Knowledge Infrastructure) until March 2021.

Main outcome(s): Insulin resistance.

Quality assessment / Risk of bias analysis: The Newcastle-Ottawa Scale was used to assess the methodological quality of the included studies, risk of bias in the selection and comparability of cohorts, and outcome (Zeng et al., 2015).

Strategy of data synthesis: A conceptual framework was constructed to guide the organization and presentation of results based on the content analysis. Besides, meta-analyses were performed.

Subgroup analysis: Subgroup analyses were performed by age distribution of study participants and region of a study conducted to explore potential sources of heterogeneity.

Sensitivity analysis: Subgroup analyses were performed by age distribution of study participants and region of a study conducted to explore potential sources of heterogeneity.

Country(ies) involved: China.

Keywords: phthalate; insulin resistance; systematic review; meta-analysis.

Contributions of each author:

Author 1 - Hui Gao.

Author 2 - Dan Chen.

Author 3 - Miao Zang. 